\title{
Perceived Roles, Benefits and Barriers of Virtual Global Health Partnership Initiatives: A Cross- Sectional Exploratory Study
}

Lisa Umphrey ( $\square$ lisa.umphrey@childrenscolorado.org )

University of Colorado Denver School of Medicine https://orcid.org/0000-0002-9502-1087

\section{George Paasi}

Mbale Clinical Research Insitute

\section{William Windsor}

Colorado School of Public Health

\section{Grace Abongo}

Mbale Clinical Research Institute

\section{Jessica Evert}

Child and Family Health International

\section{Heather Haq}

Baylor College of Medicine

\section{Elizabeth Keating}

The University of Utah School of Medicine

\section{Suet Kam Lam}

Cleveland Clinic Lerner College of Medicine of CWRU: Cleveland Clinic Lerner College of Medicine of Case Western Reserve University

\section{Megan McHenry}

Indiana University School of Medicine

\section{Carolyne Ndila}

Mbale Clinical Research Institute

\section{Charles Nwobu}

Child Family Health International

\section{Daniel Olson}

University of Colorado School of Medicine

\section{Amy Rule}

Cincinnati Children's Hospital Medical Center

\section{Reena Tam}

The University of Utah School of Medicine

\section{Peter Olupot-Olupot}

Mbale Clinical Research Institute 
Research

Keywords: Global health, partnership, virtual, pandemic

Posted Date: October 27th, 2021

DOI: https://doi.org/10.21203/rs.3.rs-979743/v1

License: (c) (i) This work is licensed under a Creative Commons Attribution 4.0 International License. Read Full License 


\section{Abstract}

\section{BACKGROUND}

Virtual global health partnership initiatives (VGHPIs) evolved rapidly during the COVID-19 pandemic to ensure partnership continuity, however the current landscape for VGHPI use and preference is unknown. This study aimed to increase understanding of GH partners' perspectives on VGHPIs.

\section{METHODS}

From 15 October to 30 November 2020, authors conducted an online, international survey using snowball sampling to document pandemic-related changes in partnership activities; preferences for VGHPIs; and perceived acceptability and barriers. Analysis stratified responses by country income classification and partnership type.

\section{RESULTS}

A total of 128 respondents described 219 partnerships. 152/219 (69\%) partnerships were transnational, $157 / 219(72 \%)$ were of $>5$ years duration, and 127/219 (60\%) included bidirectional site visits. Highincome country (HIC) partners sent significantly more learners to low- to middle-income country (LMIC) partner sites $(P<0.01)$. Participants commented on pandemic-related disruptions affecting $217 / 219$ (99\%) partnerships; 195/217 (90\%) were disruption to activities; 122/217 (56\%) to communication; 73/217 (34\%) to access to professional support; and 72/217 (33\%) to funding. Respondents indicated that VGHPIs would be important to $206 / 219$ (94\%) of their partnerships moving forward. There were overall differences in resource availability, technological capacity, and VGHPI preferences between LMIC and HIC respondents, with a statistically significant difference in VGHPI acceptability $(p<0.001)$. There was no significant difference between groups regarding VGHPIs' perceived barriers.

\section{CONCLUSIONS}

The pandemic disrupted essential partnership elements, compounding differences between LMIC and HIC partners in their resources and preferences for partnership activities. VGHPIs have the potential to bridge new and existing gaps and maximize gains, bi-directionality, and equity in partnerships during and after COVID-19.

\section{Introduction}

Global Health $(\mathrm{GH})$ is a rapidly growing field focused on advancing international and interdisciplinary healthcare (1-12). A core tenet of GH is addressing health inequities (13), and one central strategy of this approach is the implementation of Global Health Partnerships (GHPs), defined as a collaborative effort towards training, research, and capacity building $(1,14)$. The heterogeneity across diverse settings, individual preferences, and organizational interests are factors that determine the nature, duration, and future of GHPs. Moreover, the stages of development and maturity of GHPs are dependent on the 
complexity of and investment in activities undertaken locally and internationally (1-12). The focus areas of GHPs range from electives for healthcare trainees; access to resources; capacity building; technical support; professional mentorship; and collaborative research, public health, or education initiatives (1, 5-7, 14-19). Many GHPs rely heavily on unidirectional travel, with faculty and trainees from high-income countries (HIC) disproportionately visiting host sites in low- and middle-income countries (LMIC) $(1,6,7$, 14-16, 20-24). Although bidirectional exchanges of information, resources, and personnel are considered ideal $(1,2,6,7,14,15,20,22,25)$, implementing these and measuring their impact are often prohibitively expensive and complicated (6).

The COVID-19 pandemic disrupted in-person GHP activities while consequently worsening the existing inherent inequities in $\mathrm{GH}$, especially in $\operatorname{LMIC}(26,27)$. Since the start of the COVID-19 pandemic, traditional GHP operations have been affected through unprecedented travel restrictions, limitations of inperson interactions, and new standards for personal protection $(16,26,28-30)$. Challenges to traditional approaches to GHPs, including communication, bidirectional exchange of staff and learners, and inperson site visits, have continued throughout the pandemic. Beyond that, hindrances due to financial constraints, visa entry tied to COVID-19 risk or vaccination status, and GHP programing may continue to limit in-person GHP activities $(16,29)$. Concerted efforts to ensure GHP continuity are therefore paramount. Consequently, there is an urgent need for GHPs to re-examine how they will operate during the pandemic and beyond (31).

To date, there has been little research dedicated to virtual approaches to GHPs, and there are few data on ways to sustain or improve GHPs partnerships during disruptive global challenges, such as a pandemic. Guidance exists on the use of virtual education for $\mathrm{GH}$ preparation, simulation, and educational initiatives $[7,15,32-35]$, most commonly focusing on an individual activity, however less clear is how to maintain activities during the pandemic. Further, learner competencies for $\mathrm{GH}$ education exist $(1,32-35)$, but recommendations on how to reinforce competencies virtually or by distance while prioritizing the needs of partners in $\operatorname{LMIC}(16,28,36)$ are lacking. Finally, few papers compare different virtual engagement strategies or document preferences for or enablers and barriers to those strategies, particularly from the LMIC partner perspective. Virtual GHP initiatives (VGHPIs), which we define as collaborative GHP activities conducted entirely online, may inform approaches for re-organization and re-prioritization of $\mathrm{GH}$ activities.

We hypothesize that VGHPIs will improve upon and enable continuity of GHP activities during and following the COVID-19 pandemic, and that baseline data will be essential in establishing future best practice recommendations. Therefore, the impetus for this study was to generate preliminary descriptive data about VGHPIs that may inform ongoing discussions about approaches for re-organization and reprioritization of $\mathrm{GH}$ activities.

\section{Methods}

Survey Overview and Goals 
We conducted a cross-sectional, online, international survey. The survey goal was to characterize GHPs and their practices; assess changes in activities during the COVID-19 pandemic; document perceived acceptability of and barriers to VGHPIs; and enrich our understanding of alternative strategies to maintain GHPs.

\section{Survey Development}

Our team, which included members of diverse academic, non-profit, and clinical organizations, developed survey questions based on literature review and an iterative process until reaching consensus among the author group. We structured Likert scale questions to elicit baseline data on respondents' demographics; GHP characteristics; impressions of acceptability and barriers of VGHPIs; and preferences for types of VGHPIs. We included both closed-ended and free-text questions, and we allowed participants to comment on up to three GHPs. The survey and all study materials were translated from English into French and Spanish.

Expanding on previous definitions of GH $(2-4,9-12,18,26)$, for the survey, we defined a GHP as "any trans- or multinational and/or domestic collaborative health partnership that bridges geographical distance and/or resource levels to promote the health and wellbeing of people anywhere in the world." Partnerships could include headquarter or satellite sites of a single entity or represent individual organizations linked by the coalition. The collaboration could include clinical, public health, research, community, educational, and/or development work, but each were required to share a mutual focus to advance health within the capacities of their respective organizations.

Survey Target Population

Eligible participants were faculty or staff who self-identified as key members of GHPs at an organization. Ineligible participants were people not directly involved in a GHP at an organization and trainees. Because of data protection restrictions impacting European Economic Area (EEA) countries and a resultant longer ethical review period, we excluded EEA respondents.

Survey Implementation

The survey was open from 15 October to 30 November 2020. We implemented snowball sampling to access a wide range of participants in the authors' professional networks. We invited survey participation by email correspondence from each author to known focal points for GHPs (i.e., program directors, GH pathway directors, organization presidents/directors, student organization focal points, and/or clinical directors/supervisors). We also contacted listserv operators (such as one for GH residency track and fellowship directors), and GH-related social media pages (such as the twitter account for an upcoming $\mathrm{GH}$ conference). Each invitation included a request to circulate the survey link to people both within and external to a participant's organization. We sent reminder emails twice during the data collection period to encourage participation.

Data Collection 
We gathered data via an online survey created and stored via the standard encrypted online cloud platform for data collection and storage, REDCap (Vanderbilt University, http://project-redcap.org) (37). Participants received the REDCap survey link, a standard study information leaflet sheet, and study contact details. The link directed participants to a survey introduction and explanation page, and respondents who consented to participate continued to the survey. We de-identified all survey responses.

\section{Statistical Analysis}

We stratified responses by type of GHP (domestic, transnational, or both/blended) and by income status (HIC vs. LMIC) (38) of the participant's country of professional work. Using SAS software, version 9.4 (SAS Institute Inc., Cary, NC, USA), we summarized descriptive data using frequencies for dichotomous and categorical variables and measures of central tendency for continuous variables. We analyzed bivariate relationships between GHPs using chi-square tests for categorical variables. We determined statistical significance with an alpha of 0.05 . We did not implement qualitative analysis of free-text responses as we did not receive adequate qualitative data in these portions of the survey.

\section{Ethical Considerations}

We obtained institutional review board approval from the Colorado Multiple Institution Review Board (University of Colorado, Aurora, CO, USA; \#20-2099) and the Mbale Regional Referral Hospital Research Ethics Committee (Mbale, Uganda; \#MRRHREC-OUT-011/2020).

\section{Results}

A total of 134 respondents from 34 countries completed the survey (Figure 1).

Five surveys were excluded due to respondents being in the EEA and one response was excluded due to missing location. Among respondents, 115/128 (90\%) completed the survey in full, while 13/128 (10\%) responses were partial.

Table 1 shows respondent characteristics. Among respondents, 62/128 (48\%) were from LMIC while $66 / 128$ (52\%) were from HIC, and 79/128 (62\%) were from health centers/hospitals. Respondents reported that $55 / 128$ (43\%) of their organizations focused on district/county/region level work. Respondents were permitted to indicate multiple roles or specialties within the same institution. They represented a wide range of institutional roles, with 68/128 (53\%) working as professor/educators; among clinician respondents, $26 / 128(20 \%)$ worked in pediatrics, the most common specialty represented. 
Table 1

Characteristics of Respondents

\begin{tabular}{|c|c|c|}
\hline & & $\mathrm{N}(\%)$ \\
\hline \multirow[t]{5}{*}{ Respondent Region } & North America & $\begin{array}{l}65 \\
(51)\end{array}$ \\
\hline & Africa & $\begin{array}{l}39 \\
(30)\end{array}$ \\
\hline & South America & $12(9)$ \\
\hline & Asia \& Pacific & $7(6)$ \\
\hline & Middle East & $5(4)$ \\
\hline \multirow[t]{7}{*}{ Respondent's Institution Type } & Healthcare Center or Hospital & $\begin{array}{l}79 \\
(62)\end{array}$ \\
\hline & Health Profession School & $\begin{array}{l}58 \\
(45)\end{array}$ \\
\hline & Research Organization & $\begin{array}{l}26 \\
(20)\end{array}$ \\
\hline & Non-Government Organization & $\begin{array}{l}23 \\
\text { (18) }\end{array}$ \\
\hline & $\begin{array}{l}\text { Public Health or Community Service } \\
\text { Organization }\end{array}$ & $12(9)$ \\
\hline & Governmental Agency & $10(8)$ \\
\hline & Other & $7(5)$ \\
\hline \multirow[t]{5}{*}{$\begin{array}{l}\text { Geographical Reach of Respondent's } \\
\text { Organization }\end{array}$} & District/county/region & $\begin{array}{l}55 \\
(43)\end{array}$ \\
\hline & Village/town/city & $\begin{array}{l}40 \\
(31)\end{array}$ \\
\hline & National & $\begin{array}{l}38 \\
(30)\end{array}$ \\
\hline & Multi-continental & $\begin{array}{l}35 \\
(27)\end{array}$ \\
\hline & Multi-regional/country & $\begin{array}{l}21 \\
(16)\end{array}$ \\
\hline \multirow[t]{3}{*}{ Respondent role } & Professor or Educator & $\begin{array}{l}68 \\
(53)\end{array}$ \\
\hline & Clinical Staff & $\begin{array}{l}57 \\
(45)\end{array}$ \\
\hline & Researcher & $\begin{array}{l}38 \\
(30)\end{array}$ \\
\hline
\end{tabular}




\begin{tabular}{|c|c|c|}
\hline & Director/President of Organization & $\begin{array}{l}29 \\
(23)\end{array}$ \\
\hline & Administrator & $\begin{array}{l}25 \\
(20)\end{array}$ \\
\hline & Other & $11(9)$ \\
\hline \multirow[t]{7}{*}{ Respondent Clinical Discipline, if applicable } & Pediatrics & $\begin{array}{l}26 \\
(20)\end{array}$ \\
\hline & Emergency Medical Services & $11(9)$ \\
\hline & Internal Medicine & $6(5)$ \\
\hline & General Medicine & $5(4)$ \\
\hline & Surgery & $3(2)$ \\
\hline & Obstetrics/Gynecology & $2(2)$ \\
\hline & Other & $8(6)$ \\
\hline
\end{tabular}

Regarding partnerships, 77/128 (60\%) of respondents provided survey responses on $>1 \mathrm{GHP}$, and subsequently, our dataset represents 219 total GHPs. Table 2A describes characteristics of these GHPs. Of the 219 GHPs represented, 152/219 (69\%) were transnational (partnerships between different countries); $30 / 219$ (14\%) were domestic (partnerships within one country); and 17\% 37/219 (17\%) were blended (partnerships with both domestic and transnational activities). Of domestic GHPs, $26 / 30$ (87\%) described partnerships in which both members were based in a LMIC, while 4/30 (13\%) described partnerships in which both members were based within a HIC. The majority of GHPs described were $>5$ years old, with the longest partnerships between blended partnership types. The highest proportion of GHP activities reported were research and education. Domestic and blended GHPs were primarily based in LMIC, while transnational GHPs were primarily based in HIC.

Of all GHPs described, 127/219 (60\%) included bidirectional site visits while 79/219 (40\%) included unidirectional site visits. Among GHPs that included bidirectional site visits, we found that HIC partners sent significantly more learners to LMIC partner sites than LMIC partners sent to HIC sites $(36 \%$ vs. $20 \%$ respectively, $\mathrm{p}<0.001)$. Among GHPs that included unidirectional site visits, significantly more LMIC sites only hosted visitors when compared to HIC partners ( $16 \%$ vs $4 \%$ respectively, $p<0.001$ ).

Participants commented on pandemic-related disruptions to $217 / 219$ (99\%) GHPs, 97 (45\%) from the LMIC partner and 120 (55\%) from the HIC partner. Among GHPs described, 195/217 (90\%) reported a significant disruption in activities; $122 / 217$ (56\%) in communication; $73 / 217$ (34\%) in access to professional support and resources; and $72 / 217$ (33\%) in funding. Overall, more respondents from LMIC versus $\mathrm{HIC}$ reported disruption to partnership activities due to the pandemic $(\mathrm{p}=0.005)$. Of partners in LMIC, 53/97 (55\%) reported disruptions in funding, compared to only 19/120 (16\%) partners in HIC $(p<0.001)$. LMIC partners reported significantly more disruption in access to professional support and 
resources than $\mathrm{GH}$ partners in $\mathrm{HIC}(54 \%$ vs $18 \%$ respectively, $\mathrm{p}<0.001)$. Disruption in communication with partners was similar for both LMIC and HIC respondents ( $57 \%$ vs $55 \%$ respectively, $p=0.75)$.

Table 2B presents virtual activities within GHPs and future interest in VGHPIs. Before the onset of the pandemic, 84/219 (38\%) GHPs engaged in VGHPIs, most commonly occurring weekly. Respondents indicated that in the future, VGHPIs would be important for 206/219 (94\%) of their GHPs; the ways in which VGHPIs could be important, however, differed by partnership type.

Figure 2 shows the most preferred VGHPIs during the COVID-19 pandemic among GHP sites stratified by country-level income status. We found a significant difference between LMIC and HIC respondents in the distribution of all preferred VGHPIs $(p<0.001)$.

Figure 3 shows the status of technological capacity in respondents' home organization, stratified by connectivity, available infrastructure, and device accessibility. When comparing HIC and LMIC respondents, there were statistically significant differences between accessibility to a variety of resources.

Table 3 Panel A shows perceived barriers and attitudes about VHGPIs. There was no significant difference between LMIC and HIC respondents on their perceptions of barriers to VGHPIs. Regarding acceptability of VHGPIs (Table 3 Panel B), LMIC and HIC respondents differed significantly in their perceptions that they needed additional education to succeed with VGHPIs ( $48 \%$ and $14 \%$ respectively; $p<0.001)$; for preference of in-person activities versus VGHPIs ( $51 \%$ and $74 \%$, respectively; $p=0.02$ ); for requiring technical support to adequately participate in VGHPIs ( $53 \%$ and $22 \%$ respectively; $p<0.001$ ); and for ease of participating in VGHPIs $(66 \%$ and $39 \%$ respectively; $p=0.006)$. LMIC respondents were more likely to agree that virtual applications and technology were complicated compared to HIC respondents (39\% and $19 \%$, respectively; $p=0.04)$. 
Table 2

Global health partnerships - characterizations and virtual activities

\section{Partnership Type}

\begin{tabular}{llllll}
$\begin{array}{l}\text { Domestic } \\
\text { Partnerships }\end{array}$ & \multicolumn{2}{l}{$\begin{array}{l}\text { Transnational } \\
\text { Partnerships }\end{array}$} & $\begin{array}{l}\text { Blended } \\
\text { Partnerships }\end{array}$ & $\begin{array}{l}\text { P- } \\
\text { value }\end{array}$ \\
\cline { 1 - 3 } $\mathrm{N}=30$ & $(\%)$ & $\mathrm{N}=152$ & $(\%)$ & $\mathrm{N}=37 \quad(\%)$ &
\end{tabular}

Characteristics of Global Health Partnerships

\begin{tabular}{|c|c|c|c|c|c|c|c|c|}
\hline \multirow[t]{3}{*}{$\begin{array}{l}\text { Nature of } \\
\text { Partnerships }\end{array}$} & $\begin{array}{l}\text { Partnerships } \\
\text { within country* }\end{array}$ & 25 & $(83 \%)$ & 8 & $(5 \%)$ & 32 & $(86 \%)$ & $<0.001$ \\
\hline & $\begin{array}{l}\text { Partnerships } \\
\text { with LMIC- } \\
\text { based } \\
\text { organizations* }\end{array}$ & 4 & $(13 \%)$ & 115 & (76\%) & 31 & (84\%) & $<0.001$ \\
\hline & $\begin{array}{l}\text { Partnerships } \\
\text { with HIC-based } \\
\text { organizations* }\end{array}$ & 4 & $(13 \%)$ & 29 & $(19 \%)$ & 28 & (76\%) & $<0.001$ \\
\hline \multirow{3}{*}{$\begin{array}{l}\text { Length of } \\
\text { partnerships }\end{array}$} & $<5$ years & 11 & $(37 \%)$ & 42 & $(28 \%)$ & 4 & $(24 \%)$ & \multirow[t]{3}{*}{0.009} \\
\hline & $5-10$ years* & 14 & $(47 \%)$ & 50 & $(33 \%)$ & 6 & $(16 \%)$ & \\
\hline & $>10$ years & 5 & $(17 \%)$ & 60 & $(39 \%)$ & 22 & $(59 \%)$ & \\
\hline \multirow{4}{*}{$\begin{array}{l}\text { Partnership } \\
\text { activity types }\end{array}$} & Research* & 19 & $(63 \%)$ & 101 & $(66 \%)$ & 33 & $(89 \%)$ & 0.018 \\
\hline & Education & 19 & $(63 \%)$ & 120 & $(79 \%)$ & 32 & $(86 \%)$ & 0.07 \\
\hline & Clinical & 12 & $(40 \%)$ & 94 & $(62 \%)$ & 20 & $(54 \%)$ & 0.08 \\
\hline & $\begin{array}{l}\text { Community } \\
\text { Development* }\end{array}$ & 11 & $(37 \%)$ & 31 & $(20 \%)$ & 17 & $(46 \%)$ & 0.003 \\
\hline $\begin{array}{l}\text { Physical } \\
\text { interaction of } \\
\text { partnership } \\
\text { staff between } \\
\text { sites? }\end{array}$ & Yes (\%) & 23 & $(77 \%)$ & 137 & $(90 \%)$ & 32 & $(86 \%)$ & 0.12 \\
\hline \multirow{2}{*}{$\begin{array}{l}\text { Organization } \\
\text { Country Income } \\
\text { Status* }\end{array}$} & $\begin{array}{l}\text { Low/Middle } \\
\text { Income }\end{array}$ & 26 & $(87 \%)$ & 38 & $(25 \%)$ & 34 & $(92 \%)$ & \multirow[t]{2}{*}{$<0.001$} \\
\hline & High Income & 4 & $(13 \%)$ & 114 & $(75 \%)$ & 3 & $(8 \%)$ & \\
\hline
\end{tabular}

Virtual Activities within Global Health Partnerships

\begin{tabular}{lllllllll}
$\begin{array}{l}\text { Did your } \\
\text { organization } \\
\text { offer virtual } \\
\text { partnership }\end{array}$ & Yes & 12 & $(40 \%)$ & 48 & $(32 \%)$ & 24 & $(67 \%)$ & $<0.001$ \\
\cline { 2 - 9 } & No & 13 & $(43 \%)$ & 85 & $(56 \%)$ & 7 & $(19 \%)$ & \\
\cline { 2 - 7 }
\end{tabular}

activities prior 


\begin{tabular}{|c|c|c|c|c|c|c|c|c|}
\hline \multirow[t]{3}{*}{$\begin{array}{l}\text { to December } \\
2019 ?{ }^{*}\end{array}$} & & \multicolumn{7}{|c|}{ Partnership Type } \\
\hline & & \multicolumn{2}{|c|}{$\begin{array}{l}\text { Domestic } \\
\text { Partnerships }\end{array}$} & \multicolumn{2}{|c|}{$\begin{array}{l}\text { Transnational } \\
\text { Partnerships }\end{array}$} & \multicolumn{2}{|c|}{$\begin{array}{l}\text { Blended } \\
\text { Partnerships }\end{array}$} & \multirow[t]{2}{*}{$\begin{array}{l}\mathrm{P}- \\
\text { value }\end{array}$} \\
\hline & & $\mathrm{N}=30$ & $(\%)$ & $\mathrm{N}=152$ & $(\%)$ & $N=37$ & (\%) & \\
\hline \multirow{6}{*}{$\begin{array}{l}\text { What kinds of } \\
\text { virtual } \\
\text { partnership } \\
\text { activities were } \\
\text { you previously } \\
\text { engaged in? }\end{array}$} & $\begin{array}{l}\text { Planned but } \\
\text { not } \\
\text { implemented }\end{array}$ & 4 & $(13 \%)$ & 17 & $(11 \%)$ & 5 & $(14 \%)$ & \\
\hline & $\begin{array}{l}\text { Access to } \\
\text { online } \\
\text { educational } \\
\text { materials* }\end{array}$ & 5 & $(17 \%)$ & 29 & $(19 \%)$ & 15 & $(40 \%)$ & $<0.001$ \\
\hline & $\begin{array}{l}\text { Virtual face to } \\
\text { face } \\
\text { educational } \\
\text { training* }\end{array}$ & 6 & $(20 \%)$ & 31 & $(20 \%)$ & 21 & $(57 \%)$ & $<0.001$ \\
\hline & $\begin{array}{l}\text { Virtual face to } \\
\text { face clinical } \\
\text { care* }^{\star}\end{array}$ & 1 & $(3 \%)$ & 8 & $(5 \%)$ & 12 & $(32 \%)$ & $<0.001$ \\
\hline & $\begin{array}{l}\text { Virtual peer to } \\
\text { peer support }\end{array}$ & 4 & $(13 \%)$ & 20 & $(13 \%)$ & 10 & $(27 \%)$ & 0.11 \\
\hline & $\begin{array}{l}\text { Research } \\
\text { Collaborations }\end{array}$ & 5 & $(17 \%)$ & 21 & $(14 \%)$ & 9 & $(24 \%)$ & 0.29 \\
\hline \multirow{6}{*}{$\begin{array}{l}\text { How often did } \\
\text { these activities } \\
\text { occur? }\end{array}$} & Daily & 0 & $(0 \%)$ & 3 & $(6 \%)$ & 2 & $(9 \%)$ & \multirow[t]{6}{*}{0.25} \\
\hline & Weekly & 5 & $(42 \%)$ & 16 & $(34 \%)$ & 10 & $(43 \%)$ & \\
\hline & Monthly & 3 & $(25 \%)$ & 15 & $(32 \%)$ & 8 & $(35 \%)$ & \\
\hline & Quarterly & 4 & $(33 \%)$ & 5 & $(11 \%)$ & 1 & $(4 \%)$ & \\
\hline & Yearly & 0 & $(0 \%)$ & 1 & $(2 \%)$ & 2 & $(9 \%)$ & \\
\hline & $\begin{array}{l}\text { No regular } \\
\text { frequency }\end{array}$ & 0 & $(0 \%)$ & 6 & $(13 \%)$ & 0 & $(0 \%)$ & \\
\hline $\begin{array}{l}\text { Would new or } \\
\text { ongoing virtual } \\
\text { health } \\
\text { partnerships be } \\
\text { important to } \\
\text { your } \\
\text { organization? }\end{array}$ & Yes (\%) & 27 & $(90 \%)$ & 145 & $(95 \%)$ & 34 & $(100 \%)$ & 0.16 \\
\hline How? * & $\begin{array}{l}\text { Enable } \\
\text { continuity of } \\
\text { activities } \\
\text { between } \\
\text { partners }\end{array}$ & 18 & $82 \%$ & 102 & $71 \%$ & 34 & $100 \%$ & 0.002 \\
\hline
\end{tabular}




\begin{tabular}{|c|c|c|c|c|c|c|c|}
\hline & \multicolumn{7}{|c|}{ Partnership Type } \\
\hline & \multicolumn{2}{|c|}{$\begin{array}{l}\text { Domestic } \\
\text { Partnerships }\end{array}$} & \multicolumn{2}{|c|}{$\begin{array}{l}\text { Transnational } \\
\text { Partnerships }\end{array}$} & \multicolumn{2}{|c|}{$\begin{array}{l}\text { Blended } \\
\text { Partnerships }\end{array}$} & \multirow[t]{2}{*}{$\begin{array}{l}\mathrm{P} \text { - } \\
\text { value }\end{array}$} \\
\hline & $\mathrm{N}=30$ & $(\%)$ & $\mathrm{N}=152$ & $(\%)$ & $\mathrm{N}=37$ & $(\%)$ & \\
\hline $\begin{array}{l}\text { Guide planning } \\
\text { for virtual } \\
\text { collaborative } \\
\text { initiatives }\end{array}$ & 0 & $0 \%$ & 15 & $10 \%$ & 0 & $0 \%$ & \\
\hline $\begin{array}{l}\text { Allow for safer } \\
\text { partnerships }\end{array}$ & 1 & $5 \%$ & 20 & $14 \%$ & 0 & $0 \%$ & \\
\hline $\begin{array}{l}\text { Allows for } \\
\text { career } \\
\text { advancement }\end{array}$ & 3 & $14 \%$ & 6 & $4 \%$ & 0 & $0 \%$ & \\
\hline \multicolumn{8}{|c|}{ *Statistically significant distribution at $p$-value $<0.05$} \\
\hline \multicolumn{8}{|c|}{ Abbreviations: $\mathrm{LMIC}=$ low- to middle-income country; $\mathrm{HIC}=$ high income country } \\
\hline
\end{tabular}

Table 3. Perceived barriers and acceptability of virtual global health partnership initiatives (VGHPIs) among respondents 


\begin{tabular}{lllll} 
LMIC & & HIC & & p- \\
\cline { 1 - 4 } & value \\
$\mathrm{N}=61$ & $(\%)$ & $\mathrm{N}=66$ & $(\%)$ &
\end{tabular}

\section{Panel A: Perceived Barriers of VGHPIs}

\begin{tabular}{|c|c|c|c|c|c|}
\hline Lack of institution accreditation/ acknowledgement & 36 & $(60 \%)$ & 35 & $(55 \%)$ & 0.66 \\
\hline Lack of space/facilities & 37 & $(62 \%)$ & 32 & $(49 \%)$ & 0.19 \\
\hline Lack of specialty accreditation/ acknowledgement & 35 & $(58 \%)$ & 30 & $(47 \%)$ & 0.42 \\
\hline Lack of technological capacity & 41 & $(68 \%)$ & 36 & $(55 \%)$ & 0.16 \\
\hline Physical participation/ interaction requirement & 41 & $(68 \%)$ & 39 & $(61 \%)$ & 0.66 \\
\hline Time needed to train staff & 43 & $(72 \%)$ & 56 & $(86 \%)$ & 0.06 \\
\hline Cost of training & 45 & $(75 \%)$ & 42 & $(65 \%)$ & 0.44 \\
\hline Lack of institutional support & 45 & $(75 \%)$ & 48 & $(75 \%)$ & 0.58 \\
\hline Lack of mentors/local champions & 47 & $(78 \%)$ & 51 & $(78 \%)$ & 0.57 \\
\hline Lack of onsite technical support & 48 & $(80 \%)$ & 45 & $(70 \%)$ & 0.27 \\
\hline Lack of equipment for virtual education & 49 & $(82 \%)$ & 46 & $(71 \%)$ & 0.19 \\
\hline Lack of formal training curriculum & 49 & $(82 \%)$ & 49 & $(78 \%)$ & 0.51 \\
\hline \multicolumn{6}{|l|}{ Panel B: Acceptability/Usability of VGHPIs } \\
\hline \multicolumn{6}{|l|}{ Starting VGHPIs } \\
\hline I must learn many things to succeed with VGHPIs* & 28 & $(48 \%)$ & 9 & $(14 \%)$ & $<0.001$ \\
\hline $\begin{array}{l}\text { I prefer in-person activities over virtual partnership } \\
\text { activities* }\end{array}$ & 30 & $(51 \%)$ & 48 & $(74 \%)$ & 0.02 \\
\hline I would feel confident in participating in VGHPIs & 48 & $(81 \%)$ & 59 & $(91 \%)$ & 0.28 \\
\hline $\begin{array}{l}\text { I think my organization is interested in collaborating to } \\
\text { expand VGHPIs }\end{array}$ & 49 & $(83 \%)$ & 49 & $(75 \%)$ & 0.55 \\
\hline I want to implement virtual partnerships & 52 & $(88 \%)$ & 56 & $(88 \%)$ & 0.62 \\
\hline \multicolumn{6}{|l|}{ Participation in VGHPIs } \\
\hline $\begin{array}{l}\text { I need for technical support staff to adequately } \\
\text { participate* }\end{array}$ & 31 & $(53 \%)$ & 14 & $(22 \%)$ & $<0.001$ \\
\hline I think VGHPIs roll out would be smooth & 37 & $(63 \%)$ & 30 & $(46 \%)$ & 0.14 \\
\hline I anticipate continuing VGHPIs in the future & 54 & $(92 \%)$ & 63 & $(97 \%)$ & 0.19 \\
\hline
\end{tabular}




\begin{tabular}{|c|c|c|c|c|c|}
\hline & \multicolumn{2}{|l|}{ LMIC } & \multicolumn{2}{|l|}{$\mathrm{HIC}$} & \multirow{2}{*}{$\begin{array}{l}\mathrm{p}- \\
\text { value }\end{array}$} \\
\hline & $\mathrm{N}=61$ & $(\%)$ & $\mathrm{N}=66$ & $(\%)$ & \\
\hline I think VGHPIs would be awkward/uncomfortable & 12 & $(20 \%)$ & 7 & $(11 \%)$ & 0.22 \\
\hline I think VGHPIs will be inconsistent at my organization & 19 & $(32 \%)$ & 23 & $(35 \%)$ & 0.60 \\
\hline $\begin{array}{l}\text { I think virtual applications and technology are } \\
\text { complicated* }\end{array}$ & 23 & $(39 \%)$ & 12 & $(19 \%)$ & 0.04 \\
\hline I think participation in VGHPIs will be easy* & 39 & $(66 \%)$ & 25 & $(39 \%)$ & 0.01 \\
\hline I think VGHPIs can be implemented quickly & 40 & $(68 \%)$ & 34 & $(52 \%)$ & 0.19 \\
\hline I think VGHPIs would complement existing activities & 56 & $(95 \%)$ & 61 & $(94 \%)$ & 0.35 \\
\hline $\begin{array}{l}\text { *Statistically significant distribution at p-value }<0.05 \\
\text { Abbreviations: VGHPI=Virtual Global Health Partnershi } \\
\text { HIC=High Income Country }\end{array}$ & tiati & $A I C=$ & $v / M i$ & ncor & ountry; \\
\hline
\end{tabular}

\section{Discussion}

To our knowledge, our study is the first to explore the role of VGHPIs and their perceived benefits and barriers for the resilience of GHPs during the COVID-19 pandemic. Further, our survey is the first to query current LMIC and HIC members of GHPs to document initial preferences for and interest in VGHPIs. Although our data are descriptive only, our findings contribute critical information to advance previous discussions about supporting partners in LMIC during global challenges, such as epidemics or crises [10, 43]. Additionally our findings add a real-world perspective to recent discussions about shifting GHP activities virtually $(16,28,36,39)$, addressing LMIC partner needs thoughtfully (30), addressing virtual education needs within certain specialties $(19,26,29)$, and proposing virtual programming relevant only in $\operatorname{HIC}(40,41)$.

Among our 128 respondents from 34 countries, there were a wide range of institutions and clinical specialties represented. Participants shared details of up to three of their GHPs, which allowed for an expanded dataset within a relatively limited participant group. The GHPs described in this study are like those in previous studies $(1,6,7,14-16,20-25)$, and our data offer insights into VGHPI considerations for similar GHPs. A few GHPs in our dataset represented domestic LMIC/LMIC or HIC/HIC partnerships, "global local" pairings whose unique needs should be considered during implementation of VGHPIs ( 1,2 , 8). Most partnerships were bidirectional, but the reported exchanges, whether bidirectional or unidirectional, were mainly from the HIC to the LMIC partner. Previously described successful bidirectional and collaborative initiatives during crises $(15,42)$ may be just as (or more) easily done virtually and favor the needs of the LMIC partner. Such transferable activities include opening access to educational resources; connecting subspecialists from HIC to LMIC sites; assisting LMIC faculty with 
grant writing and budget preparation; assisting LMIC trainees with residency application or entry examination preparation; offloading administrative tasks from the LMIC partner to HIC partner; and advocating for funds to improve LMIC partners' administrative, office and technological capacity. VGHPIs provide an opportunity for complementing and coordinating efforts in GHPs, a tenet of ethical GH practices(43), more efficiently than ever before.

Not surprisingly, only a minority of GHPs were engaged in VGHPIs before the pandemic, often with a weekly frequency. Thus, future studies looking at ideal activity frequency and ongoing activity preferences will be helpful in providing best practice recommendations. Interestingly, the respondents reported that VGHPIs would be important to the vast majority $(206 / 219,94 \%)$ of their GHPs moving forward. The significant differences in opinion about how VGHPIs would be important for domestic, transnational, and blended GHPs will be key discussion points for partnerships looking to incorporate or expand VGHPIs. Although we only inquired about four ways in which VGHPIs may be important (enabling continuity of activities vs usefulness in guiding planning vs increasing safety of partnerships vs allowing for career advancement), our findings suggest that partners' priorities and needs may not be aligned, and shared priorities should not be assumed. Likewise, data showing the significant differences between HIC and LMIC partners regarding preferred types of VGHPIs, most specifically in terms of access to online materials (preferred by LMIC more than HIC respondents) and valuing virtual face-to-face trainings (preferred by HIC more than LMIC respondents), are telling. Because HIC and LMIC partners seem to value different components of VGHPIs, these notable differences should prompt ongoing and intentional discussions to ensure all parties are mutually benefitting from VGHPI implementation and roll out.

Our data show ongoing discrepancies between resource access and allocation that worsened since the pandemic began. For example, most respondents reported pandemic-related disruptions in communication at their GHP sites, but disruptions in funding, partnership activities, and access to professional support and resources were significantly more disruptive for LMIC compared to HIC respondents. This must be taken into consideration for future emergency responses and in how VGHPIs are structured and planned from baseline.

Regarding barriers to VGHPIs, although there were no significant differences between LMIC and HIC respondents on perceived barriers to VGHPIs, the agreement between respondents could counter assumptions that members of GHPs may make about each other. The time needed for training and the lack of training curriculum were among the most frequently reported barriers by GHP sites. This suggests underlying healthcare system challenges in enabling continuous professional education within the GHPs. Regarding technological capacity, our findings agree with previous studies $(1,8,14,36,44-46)$ that suggest a lack of internet connectivity is a severe concern for GHPs, with important implications for VGHPIs. We found that LMIC partners reported less access to wireless internet, less trainee access to organization-owned hardware, poorer cellular phone service, and less access to physical spaces like meeting and simulation facilities. However, both HIC and LMIC respondents had reliable access to personal smartphones, to organization-owned technology, and video-conferencing services. Considering the technological capacity within GHPs and possibly investing into communication infrastructure will be 
critical to ensure successful virtual engagement. Funding for in-person activities could be shifted towards resources that improve internet connectivity at LMIC partner-sites to address this challenge.

Between the LMIC and HIC partners, there were several key differences in opinion about VGHPI acceptability. Significantly more respondents in LMIC compared to HIC reported they would need to learn many things to succeed with VGHPIs and require technical support to fully participate in VGHPIs. This need for education and support must be considered moving forward to ensure the needs of LMIC partners are adequately heard and met. Interestingly, more LMIC respondents reported that participating in VGHPIs would be easy, but that virtual applications and technology are complicated. This perhaps reflects the difference between using technology (something many LMIC partners are accustomed to as the hosting partner in a GHP) versus reliable access to technology (reflecting challenges in technological capacity), which indicates that reliable access must be accompanied by reliable training. This could be further examined in future studies.

Our study had several limitations. First, the pandemic likely hindered invited respondents from participating. We actively reached out to our professional networks to mitigate this challenge, but in doing so may have favored respondents from partnerships with greater resources. Second, as an initial exploratory survey, we pursued convenience sampling, which meant it was not possible to document response rate to the survey. Third, we chose not to include participants from the EEA due to lengthy ethical review processes to meet European data protection requirements, an issue we plan to address in future surveys. Fourth, our paper presents only descriptive data given that we received inadequate freeresponse data for a qualitative analysis. Finally, partial survey response may have affected results, though this was only $10 \%$ of the respondents; the pattern was determined to be missingness completely at random and did not disrupt results. Despite the limitations, we believe we gained valuable insight into a wide variety of GHPs at a key moment during the COVID-19 pandemic. Further, we approached this survey as a first step to inform future mixed-method and qualitative work about VGHPIs, helping to direct further study.

\section{Conclusion}

GHPs were significantly disrupted by the COVID-19 pandemic, in particular their funding, communication, resource access, and activities, but the ways in which these disruptions affected LMIC versus HIC partners were disparate. Our survey of GHPs in both HIC and LMIC identified a strong enthusiasm for VGHPIs despite several key barriers. VGHPIs may bridge existing gaps and maximize gains of GHPs for enhanced bidirectionality, transparency, and equity in GHPs. Future study is needed to measure and guide best practices in this rapidly developing landscape.

Acronyms

$\mathrm{EEA}=$ European Economic Area

$\mathrm{GH}=$ global health 
GHP = global health partnership

HIC = high-income country

LMIC = low- to middle-income country

VGHPI = virtual global health partnership initiative

\section{Abbreviations}

$\mathrm{EEA}=$ European Economic Area

$\mathrm{GH}=$ global health

$\mathrm{GHP}=$ global health partnership

HIC = high-income country

LMIC = low- to middle-income country

VGHPI = virtual global health partnership initiative

\section{Declarations}

\section{ETHICAL APRPOVAL and CONSENT TO PARTICIPATE}

We obtained institutional review board approval from the Colorado Multiple Institution Review Board (University of Colorado, Aurora, CO, USA; protocol \#20-2099, exempted 27 Aug 2020) and the Mbale Regional Referral Hospital Research Ethics Committee (MRRH-REC)/, Mbale, Uganda; protocol \#MRRHREC-OUT-011/2020, accepted 30 Sept 2020). All participants provided consent to complete the study's survey as per ethical review board requirements.

\section{CONSENT FOR PUBLICATION}

Non-applicable

\section{AVAILABILITY OF DATA AND MATERIALS}

The datasets used and/or analyzed during the current study are available from the corresponding author on reasonable request.

\section{COMPETING INTERESTS}

The authors declare that they have no competing interests. 
ATLAS International, Inc. provided funding for ethical review in Uganda.

\section{AUTHORS' CONTRIBUTIONS}

LU conceived project idea. LU and GP created initial project proposal and survey drafts

All authors contributed to survey edits and finalization and survey circulation and data collection. LU, CN, GA, and WW conducted survey and database creation and management. WW, LU, GP, and POO performed data analysis. LU, WW, GP, and POO created original manuscript draft. All authors contributed to manuscript editing and finalization.

\section{ACKNOWLEDGMENTS}

The authors wish to thank Viviane Leuche, Geoffrey Winstanley, Mery Paz Alvarado, Grace Artega, Risha Moskalewicz, and Stephanie Perez Kerkvliet for their assistance with French and Spanish translations; the Midwest Consortium of Global Child Health Educators; and those who participated in the survey.

\section{Authors information (optional)}

Non-applicable

\section{References}

1. Steenhoff AP, Crouse HL, Lukolyo H, Larson CP, Howard C, Mazhani L, et al. Partnerships for Global Child Health. Pediatrics. 2017;140(4).

2. Rowthorn V. Global/Local: What Does It Mean for Global Health Educators and How Do We Do It? Ann Glob Health. 2015;81(5):593-601.

3. Beaglehole R, Bonita R. What is global health? Glob Health Action. 2010;3.

4. Koplan JP, Bond TC, Merson MH, Reddy KS, Rodriguez MH, Sewankambo NK, et al. Towards a common definition of global health. Lancet. 2009;373(9679):1993-5.

5. Fried LP, Bentley ME, Buekens P, Burke DS, Frenk JJ, Klag MJ, et al. Global health is public health. Lancet. 2010;375(9714):535-7.

6. Pitt MB, Gladding SP, Majinge CR, Butteris SM. Making Global Health Rotations a Two-Way Street: A Model for Hosting International Residents. Glob Pediatr Health. 2016;3:2333794X16630671.

7. Batra M, Pitt MB, St Clair NE, Butteris SM. Global Health and Pediatric Education: Opportunities and Challenges. Adv Pediatr. 2018;65(1):71-87.

8. Editors MR. Closing the "Know-Do" Gap: eHealth Strategies for the Global South. MEDICC Rev. 2008;10(1):3.

9. Abimbola S. On the meaning of global health and the role of global health journals. Int Health. 2018;10(2):63-5. 
10. Campbell RM, Pleic M, Connolly H. The importance of a common global health definition: How Canada's definition influences its strategic direction in global health. J Glob Health. 2012;2(1):010301.

11. Frenk J, Gomez-Dantes O, Moon S. From sovereignty to solidarity: a renewed concept of global health for an era of complex interdependence. Lancet. 2014;383(9911):94-7.

12. King NB, Koski A. Defining global health as public health somewhere else. BMJ Glob Health. 2020;5(1):e002172.

13. Farmer PE, Furin JJ, Katz JT. Global health equity. Lancet. 2004;363(9423):1832.

14. Rees CA, Keating EM, Dearden KA, Haq H, Robison JA, Kazembe PN, et al. Improving Pediatric Academic Global Health Collaborative Research and Agenda Setting: A Mixed-Methods Study. Am J Trop Med Hyg. 2020;102(3):649-57.

15. McQuilkin P, Marshall RE, Niescierenko M, Tubman VN, Olson BG, Staton D, et al. A Successful US Academic Collaborative Supporting Medical Education in a Postconflict Setting. Glob Pediatr Health. 2014;1:2333794X14563383.

16. Kalbarczyk A, Harrison M, Sanguineti MCD, Wachira J, Guzman CAF, Hansoti B. Practical and Ethical Solutions for Remote Applied Learning Experiences in Global Health. Ann Glob Health. 2020;86(1):103.

17. Evert J. Teaching corner: child family health international : the ethics of asset-based global health education programs. J Bioeth Inq. 2015;12(1):63-7.

18. Godoy-Ruiz P, Cole DC, Lenters L, McKenzie K. Developing collaborative approaches to international research: Perspectives of new global health researchers. Glob Public Health. 2016;11(3):253-75.

19. Lepard JR, Akbari SHA, Haji F, Davis MC, Harkness W, Johnston JM. The initial experience of InterSurgeon: an online platform to facilitate global neurosurgical partnerships. Neurosurg Focus. 2020;48(3):E15.

20. Bozinoff N, Dorman KP, Kerr D, Roebbelen E, Rogers E, Hunter A, et al. Toward reciprocity: host supervisor perspectives on international medical electives. Med Educ. 2014;48(4):397-404.

21. Butteris SM, Schubert CJ, Batra M, Coller RJ, Garfunkel LC, Monticalvo D, et al. Global Health Education in US Pediatric Residency Programs. Pediatrics. 2015;136(3):458-65.

22. Hudspeth JC, Rabin TL, Dreifuss BA, Schaaf M, Lipnick MS, Russ CM, et al. Reconfiguring a One-Way Street: A Position Paper on Why and How to Improve Equity in Global Physician Training. Acad Med. 2019;94(4):482-9.

23. Lukolyo H, Rees CA, Keating EM, Swamy P, Schutze GE, Marton S, et al. Perceptions and Expectations of Host Country Preceptors of Short-Term Learners at Four Clinical Sites in Sub-Saharan Africa. Acad Pediatr. 2016;16(4):387-93.

24. Pitt MB, Gladding SP, Suchdev PS, Howard CR. Pediatric Global Health Education: Past, Present, and Future. JAMA Pediatr. 2016;170(1):78-84. 
25. Arora G, Russ C, Batra M, Butteris SM, Watts J, Pitt MB. Bidirectional Exchange in Global Health: Moving Toward True Global Health Partnership. Am J Trop Med Hyg. 2017;97(1):6-9.

26. Karim N, Rybarczyk MM, Jacquet GA, Pousson A, Aluisio AR, Bilal S, et al. COVID-19 Pandemic Prompts a Paradigm Shift in Global Emergency Medicine: Multidirectional Education and Remote Collaboration. AEM Educ Train. 2021;5(1):79-90.

27. Shamasunder S, Holmes SM, Goronga T, Carrasco H, Katz E, Frankfurter R, et al. COVID-19 reveals weak health systems by design: Why we must re-make global health in this historic moment. Glob Public Health. 2020;15(7):1083-9.

28. Amerson R. Striving to Meet Global Health Competencies Without Study Abroad. J Transcult Nurs. 2021;32(2):180-5.

29. Chatziralli I, Ventura CV, Touhami S, Reynolds R, Nassisi M, Weinberg T, et al. Transforming ophthalmic education into virtual learning during COVID-19 pandemic: a global perspective. Eye (Lond). 2020:1-8.

30. McHenry MS, Tam RP, Nafiseh AA, Etling MA, Barnes AE, Rule ARL, et al. Global Health Partnerships During the COVID-19 Pandemic: Perspectives and Insights from International Partners. Am J Trop Med Hyg. 2021.

31. Conway JHMRGOCW. Issues concerning overseas travel that international educators must consider in the coming months (opinion). 2021.

32. Global Health Education Competencies Toolkit | Consortium of Universities for Global Health: @CUGHnews; 2018 [Available from: https://www.cugh.org/online-tools/competencies-toolkit/.

33. Ahrens K, Stapleton FB, Batra M. The university of washington pediatric residency program experience in global health and community health and advocacy. Virtual Mentor. 2010;12(3):184-9.

34. DeCamp M, Rodriguez J, Hecht S, Barry M, Sugarman J. An ethics curriculum for short-term global health trainees. Global Health. 2013;9:5.

35. Shah S, Lin HC, Loh LC. A Comprehensive Framework to Optimize Short-Term Experiences in Global Health (STEGH). Global Health. 2019;15(1):27.

36. Ambrose M, Murray L, Handoyo NE, Tunggal D, Cooling N. Learning global health: a pilot study of an online collaborative intercultural peer group activity involving medical students in Australia and Indonesia. BMC Med Educ. 2017;17(1):10.

37. Harris PA, Taylor R, Thielke R, Payne J, Gonzalez N, Conde JG. Research electronic data capture (REDCap)-a metadata-driven methodology and workflow process for providing translational research informatics support. J Biomed Inform. 2009;42(2):377-81.

38. World Bank Country and Lending Groups â World Bank Data Help Desk 2021 [Available from: https://datahelpdesk.worldbank.org/knowledgebase/articles/906519-world-bank-country-andlending-groups.

39. Hou L, Mehta SD, Christian E, Joyce B, Lesi O, Anorlu R, et al. Impact of the COVID-19 pandemic on global health research training and education. J Glob Health. 2020;10(2):020366. 
40. Chick RC, Clifton GT, Peace KM, Propper BW, Hale DF, Alseidi AA, et al. Using Technology to Maintain the Education of Residents During the COVID-19 Pandemic. J Surg Educ. 2020;77(4):729-32.

41. Remtulla R. The Present and Future Applications of Technology in Adapting Medical Education Amidst the COVID-19 Pandemic. JMIR Med Educ. 2020;6(2):e20190.

42. McQuilkin PA, Niescierenko M, Beddoe AM, Goentzel J, Graham EA, Henwood PC, et al. Academic Medical Support to the Ebola Virus Disease Outbreak in Liberia. Acad Med. 2017;92(12):1674-9.

43. Crump JA, Sugarman J, Working Group on Ethics Guidelines for Global Health T. Ethics and best practice guidelines for training experiences in global health. Am J Trop Med Hyg. 2010;83(6):117882.

44. Barteit S, Sie A, Ye M, Depoux A, Louis VR, Sauerborn R. Lessons learned on teaching a global audience with massive open online courses (MOOCs) on health impacts of climate change: a commentary. Global Health. 2019;15(1):52.

45. Brzoska P, Akgun S, Antia BE, Thankappan KR, Nayar KR, Razum O. Enhancing an International Perspective in Public Health Teaching through Formalized University Partnerships. Front Public Health. 2017;5:36.

46. Grant J. Distance learning: A response to overcome current challenges in medical education? Towards Unity for Health: Coordinating changes in health services and health professions practice and education. 2001;4.

\section{Figures}




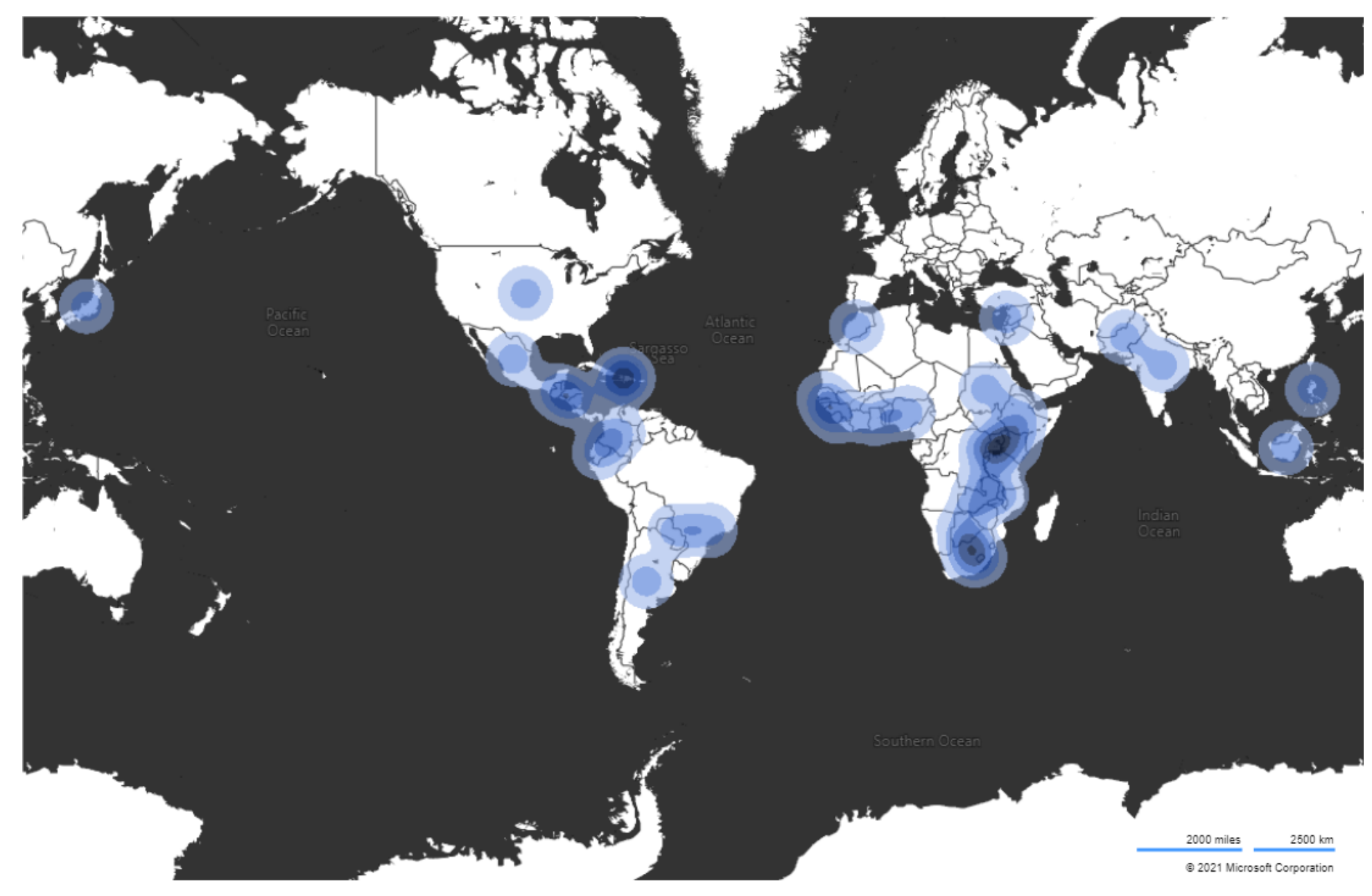

Figure 1

Distribution of Respondents to the Virtual Global Health Partnership Initiatives during COVID-19 Survey 


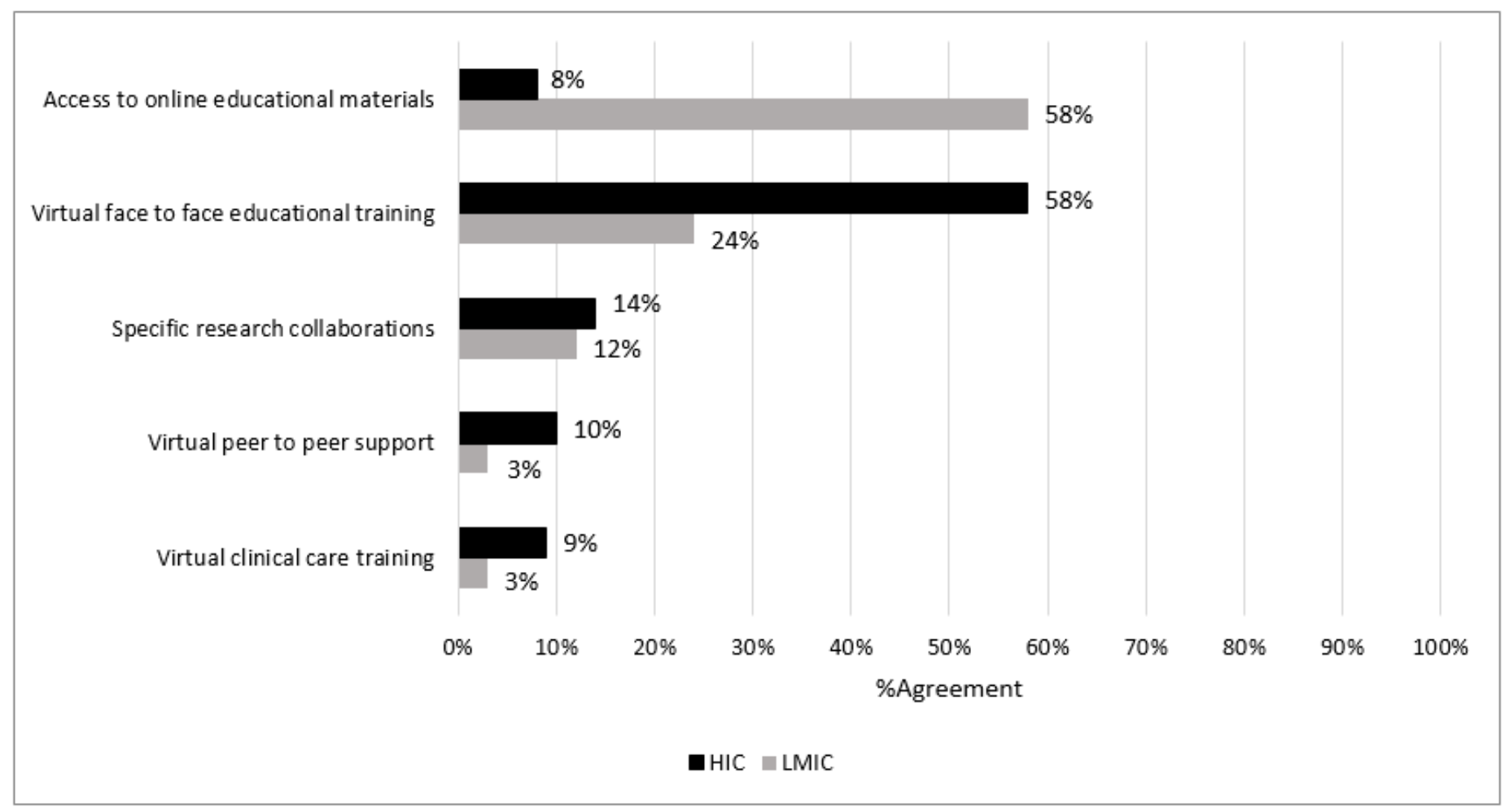

The distribution of preferred VGHPIs by country income status was statistically significant at $p<0.001$

Abbreviations: LMIC=Low/Middle Income Country; HIC=High Income Country

\section{Figure 2}

Most highly preferred virtual global Health partnership initiatives (VGHPIs) during the COVID-19 pandemic 
Figure 2, Panel A: Connectivity

Reliable Wi-Fi/internet connection in office/administrative areas*

Reliable WIFI/internet access to faculty/staff*

Reliable WIFI/internet access to trainees*

Reliable Wi-Fi in clinical care areas*
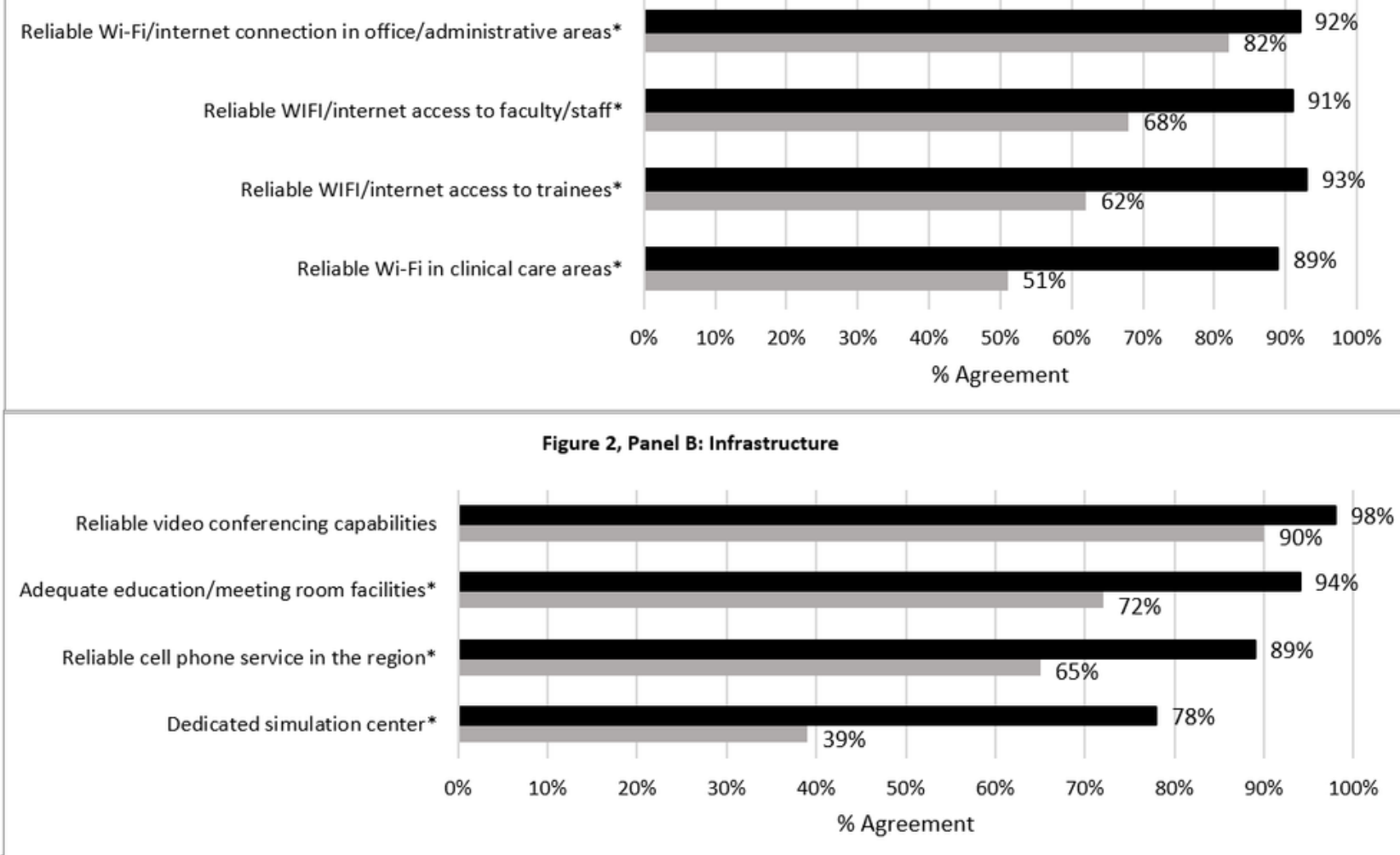

Figure 2, Panel C: Access to Devices

Faculty/staff access to an institution-owned smart phone

Trainee access to an institution-owned computer*

Faculty/staff access to an institution-owned computer

Trainee access to personal smart phone

Faculty/staff access to personal smart phone.

Trainee access to an institution-owned smart phone*

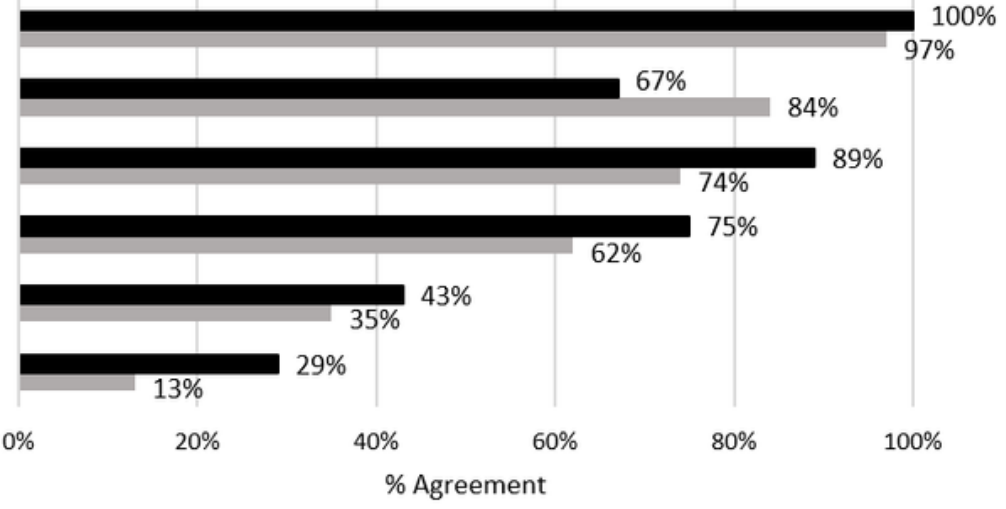

HIC

*Statistically significant distribution at p-value $<0.05$

Abbreviations: LMIC=Low/Middle Income Country; HIC=High Income Country

\section{Figure 3}

Technological capacity for virtual global health partnership initiatives (VGHPIs) among respondents 